\title{
Olive Leaf Extracts Protect Cardiomyocytes against 4-Hydroxynonenal-Induced Toxicity In Vitro: Comparison with Oleuropein, Hydroxytyrosol, and Quercetin
}

Authors

Affiliations
Elif Burcu Bali ${ }^{1 *}$, Volkan Ergin ${ }^{2 *}$, Lucia Rackova ${ }^{3}$, Oğuz Bayraktar ${ }^{4}$, Nurgün Küçükboyacı ${ }^{5}$, Çimen Karasu ${ }^{1}$

The affiliations are listed at the end of the article

\author{
Key words \\ - Olea europaea L. \\ - Oleaceae \\ - olive leaf extract \\ - cardiomyocytes \\ - 4-hydroxynonenal \\ - oleuropein \\ - hydroxytyrosol \\ - quercetin
}

\section{received January 19, 2014 \\ revised May 14, 2014 \\ accepted June 16, 2014}

Bibliography

DOI http://dx.doi.org/

10.1055/s-0034-1382881

Published online

Planta Med $\odot$ Georg Thieme

Verlag KG Stuttgart · New York .

ISSN 0032-0943

Correspondence

Prof. Dr. Çimen Karasu

Cellular Stress Response and

Signal Transduction Research

Laboratory

Department of Medical

Pharmacology

Faculty of Medicine

Gazi University

06500, Beşevler, Ankara

Turkey

Phone: + 903122026921

Fax: + 903122124647

cimenkrs@gmail.com

\section{Abstract}

$\nabla$

Olive (Olea europaea) leaf, an important traditional herbal medicine, displays cardioprotection that may be related to the cellular redox modulating effects of its polyphenolic constituents. This study was undertaken to investigate the protective effect of the ethanolic and methanolic extracts of olive leaves compared to the effects of oleuropein, hydroxytyrosol, and quercetin as a positive standard in a carbonyl compound (4-hydroxynonenal)-induced model of oxidative damage to rat cardiomyocytes (H9c2). Cell viability was detected by the MTT assay; reactive oxygen species production was assessed by the $2^{\prime}, 7^{\prime}$-dichlorodihydrofluorescein diacetate method, and the mitochondrial membrane potential was determined using a JC-1 dye kit. Phospho-Hsp27 (Ser82), phospho-MAPKAPK-2 (Thr334), phospho-c-Jun (Ser73), cleaved-caspase-3 (cl-CASP3) (Asp175), and phospho-SAPK/JNK (Thr183/Tyr185) were measured by Western blotting. The ethanolic and methanolic extracts of olive leaves inhibited 4-hydroxynonenal-induced apoptosis, characterized by increased reactive oxygen species production, impaired viability $\left(\mathrm{LD}_{50}: 25 \mu \mathrm{M}\right)$, mitochondrial dysfunction, and activation of pro-apoptotic clCASP3. The ethanolic and methanolic extracts of olive leaves also inhibited 4-hydroxynonenal-induced phosphorylation of stress-activated transcription factors, and the effects of extracts on $\mathrm{p}$ SAPK/JNK, p-Hsp27, and p-MAPKAPK-2 were found to be concentration-dependent and comparable with oleuropein, hydroxytyrosol, and quercetin. While the methanolic extract downregulated 4-hydroxynonenal-induced p-MAPKAPK-2 and p-c-Jun more than the ethanolic extract, it exerted a less inhibitory effect than the ethanolic extract on 4-hydroxynonenal-induced

\footnotetext{
* Both of these authors contributed equally to this study.
}

p-SAPK/JNK and p-Hsp27. cl-CASP3 and p-Hsp27 were attenuated, especially by quercetin. Experiments showed a predominant reactive oxygen species inhibitory and mitochondrial protecting ability at a concentration of $1-10 \mu \mathrm{g} / \mathrm{mL}$ of each extract, oleuropein, hydroxytyrosol, and quercetin. The ethanolic extract of olive leaves, which contains larger amounts of oleuropein, hydroxytyrosol, verbascoside, luteolin, and quercetin (by HPLC) than the methanolic one, has more protecting ability on cardiomyocyte viability than the methanolic extract or each phenolic compound against 4-hydroxynonenal-induced carbonyl stress and toxicity.

\section{Abbreviations \\ $\nabla$ \\ cl-CASP3: cleaved caspase-3 \\ DCF: $\quad 2$ ',7'-dichlorofluorescein}

DCFH2-DA: 2',7'-dichlorodihydrofluorescein diacetate

$\Delta \Psi \mathrm{m}$ : mitochondrial membrane potential

HNE: 4-hydroxynonenal

Hsp27: heat shock protein 27

H9c2: embryonic rat heart-derived myogenic cell line

JC-1: $\quad 5,5^{\prime}, 6,6^{\prime}$-tetrachloro-1,1',3,3'-tetraethylbenzimidazolo carbocyanine iodide

MAPKAPK-2: MAP kinase-activated protein kinase 2

MTT: 3-(4,5-dimethylthiazol-2-yl)-2,5diphenyl tetrazolium bromide

OLE-1: ethanolic extract of olive leaves

OLE-2: methanolic extract of olive leaves

PMSF: phenylmethanesulfonyl fluoride

ROS: $\quad$ reactive oxygen species

SAPK/JNK: stress-activated protein kinase/ c-jun $\mathrm{N}$-terminal kinases 


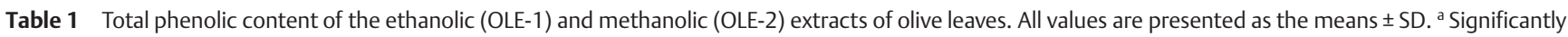
different from OLE-1 $(p<0.05)$; Student's $t$-test. GAE: gallic acid equivalent.

$\begin{array}{llllr}\text { Sample }(\mathbf{1 ~ m g} / \mathbf{m L}) & \text { Absorbance } & \text { SD } & \text { GAE }(\mathbf{m g} / \mathbf{m L}) & \% \\ \text { OLE-1 } & 0.78 & 0.03 & 0.14 & 14.1 \\ \text { OLE-2 } & 0.55^{\mathrm{a}} & 0.01 & 0.09 & 8.7\end{array}$

\begin{tabular}{|c|c|c|c|}
\hline Extract & Concentration (mg/mL) & $\%$ & Retention time (min) HPLC \\
\hline \multicolumn{4}{|c|}{ Oleuropein } \\
\hline OLE-1 & 2.047 & 20.47 & 21.740 \\
\hline OLE-2 & $0.354^{\mathrm{a}}$ & 3.54 & 21.753 \\
\hline \multicolumn{4}{|c|}{ Hydroxytyrosol } \\
\hline$>$ OLE-1 & 0.040 & 0.40 & 5.598 \\
\hline$>$ OLE-2 & $0.023^{\mathrm{a}}$ & 0.23 & 5.573 \\
\hline \multicolumn{4}{|c|}{ Verbascoside } \\
\hline - OLE-1 & 0.060 & 0.60 & 16.968 \\
\hline - OLE-2 & $0.035^{\mathrm{a}}$ & 0.35 & 16.979 \\
\hline \multicolumn{4}{|l|}{ Quercetin } \\
\hline - OLE-1 & 0.039 & 0.39 & 25.051 \\
\hline > OLE-2 & $0.003^{a}$ & 0.03 & 25.737 \\
\hline \multicolumn{4}{|l|}{ Luteolin } \\
\hline D OLE-1 & 0.167 & 1.67 & 26.186 \\
\hline OLE-2 & $0.021^{\mathrm{a}}$ & 0.21 & 26.543 \\
\hline
\end{tabular}

Table 2 Major phenolic compounds of the ethanolic (OLE-1) and methanolic (OLE-2) extracts of olive leaves. ${ }^{\text {a }}$ Significantly different from OLE-1 $(p<0.05)$; Student's $t$ test.

\section{Introduction \\ $\nabla$}

The interactions of proteins with ROS may result in numerous post-translational, reversible, or irreversible modifications, leading to a change in structure and/or function of the oxidized protein. 4-hydroxynonenal (HNE) is a major active aldehyde formed by lipid peroxidation reactions, which can react readily with histidine, cysteine, or lysine residues of proteins, leading to the formation of stable Michael adducts with a hemiacetal structure [1$3]$. The modifications of proteins by reactive aldehydes act as signaling molecules and play multiple physiological functions; however, their excessive production with irreversible concentrations may contribute to cytopathological results including impaired signaling, RNA and DNA damage, cell cycle arrest, and apoptosis [4-6]. HNE is involved in various disease states including atherosclerosis [5], diabetes [7,8], and cardiac problems [9,10]. In cultured cardiomyocytes, HNE has been shown to mediate hypoxia/ reoxygenation-induced injury and cell death inhibited by antioxidant treatment [11]. Despite the significance of HNE or carbonyl stress in cell survival or the death process, the effects of HNE incubation in cardiomyocytes remain uninvestigated. The aim of this study was to determine the mechanism of carbonyl (HNE)induced apoptotic signaling and cardiotoxicity in H9c2 cells.

Olive leaves contain several groups of constituents, including polyphenols, flavonoids, flavones, iridoids, and carbohydrates [12-14]. Oleuropein, the major phenolic secoiridoid in Olea europaea L. (Oleaceae), has been characterized as a powerful antioxidant in in vitro $[14,15]$ and in vivo [16] experiments. Olive leaves or oleuropein have been shown to attenuate cardiac, hepatic, and metabolic changes in high-carbohydrate-, high-fat-fed rats [16, 17] and protect heart function against ischemia and reperfusion [18]. In recent studies, we showed that the ethanolic extracts of olive leaves, oleuropein, or hydroxytyrosol exhibited the protective and regenerative effects on redox balance and related signaling pathways in insulin releasing pancreatic $\beta$-cells exposed to $\mathrm{H}_{2} \mathrm{O}_{2}$ [19] or a cytokine cocktail [20]. Thus, in this study, we sought to explore if olive leaf extracts or polyphenolic com- pounds (oleuropein, hydroxytyrosol and quercetin) can protect H9c2 cardiomyocytes in vitro against carbonyl (HNE) stress-induced toxicity and apoptosis.

\section{Results}

$\nabla$

The amount of total phenolic content of olive leaf extracts determined using the gallic acid calibration curve (data not showed) was found to be higher in OLE-1 (ethanolic extract) than OLE-2 (methanolic extract) ( Table 1). Larger amounts of oleuropein, which is the major phenolic constituent of olive leaves [12-14, $19,20]$, were detected in both extracts than the amounts of other phenolic constituents. The quantity of oleuropein was found to be more in OLE-1 than in OLE-2. OLE-2 also contains less hydroxytyrosol, verbascoside, luteolin, and quercetin than OLE-1 ( Table 2). High-performance liquid chromatography (HPLC) chromatograms of the ethanolic and methanolic extracts of olive leaves are demonstrated in $\bullet$ Fig. 1.

$\mathrm{H} 9 \mathrm{c} 2$ cells were preincubated with increasing concentrations $(0.1,10,25$, and $50 \mu \mathrm{g} / \mathrm{mL})$ of each extract, phenolic compound, or standard quercetin for $24 \mathrm{~h}$. At $0.1,10,25$, and $50 \mu \mathrm{g} / \mathrm{mL}$ concentration ranges, cell viability was $87.07 \pm 2.01 \%$, $91.16 \pm 8.09 \%$, $85.89 \pm 6.81 \%$, and $77.49 \pm 6.87 \%$ for OLE- 1 , respectively; $96.02 \pm$ $1.88 \%, 96.02 \pm 2.75 \%, 96.02 \pm 0.944 \%$, and $88.76 \pm 4.30 \%$ for OLE2 , respectively; $80.54 \pm 2.82 \%, 81.26 \pm 3.07 \%, 83.50 \pm 1.36 \%$, and $76.20 \pm 2.87 \%$ for oleuropein, respectively; $68.55 \pm 4.47 \%, 68.22 \pm$ $7.50 \%, 74.88 \pm 2.77 \%, 66.11 \pm 10.32 \%$, and $45.93 \pm 2.74 \%$ for hydroxytyrosol, respectively; and $89.0 \pm 6.80 \%$, $92.0 \pm 5.50 \%$, $89.70 \pm 3.40 \%$, and $95.20 \pm 11.30 \%$ for quercetin, respectively. According to these findings, the minimal effective two concentrations of the extracts, phenolic compounds, and the standard quercetin were selected for testing their protective effect on HNE toxicity by the MTT method.

- Fig. 2 shows that HNE $(0-50 \mu \mathrm{M})$ induced inhibition on the viability of $\mathrm{H} 9 \mathrm{c} 2$ cells in a dose-dependent manner. The $\mathrm{IC}_{50}$ value of $25 \mu \mathrm{M}$ of HNE was obtained at $6 \mathrm{~h}$. We used non-toxic doses 


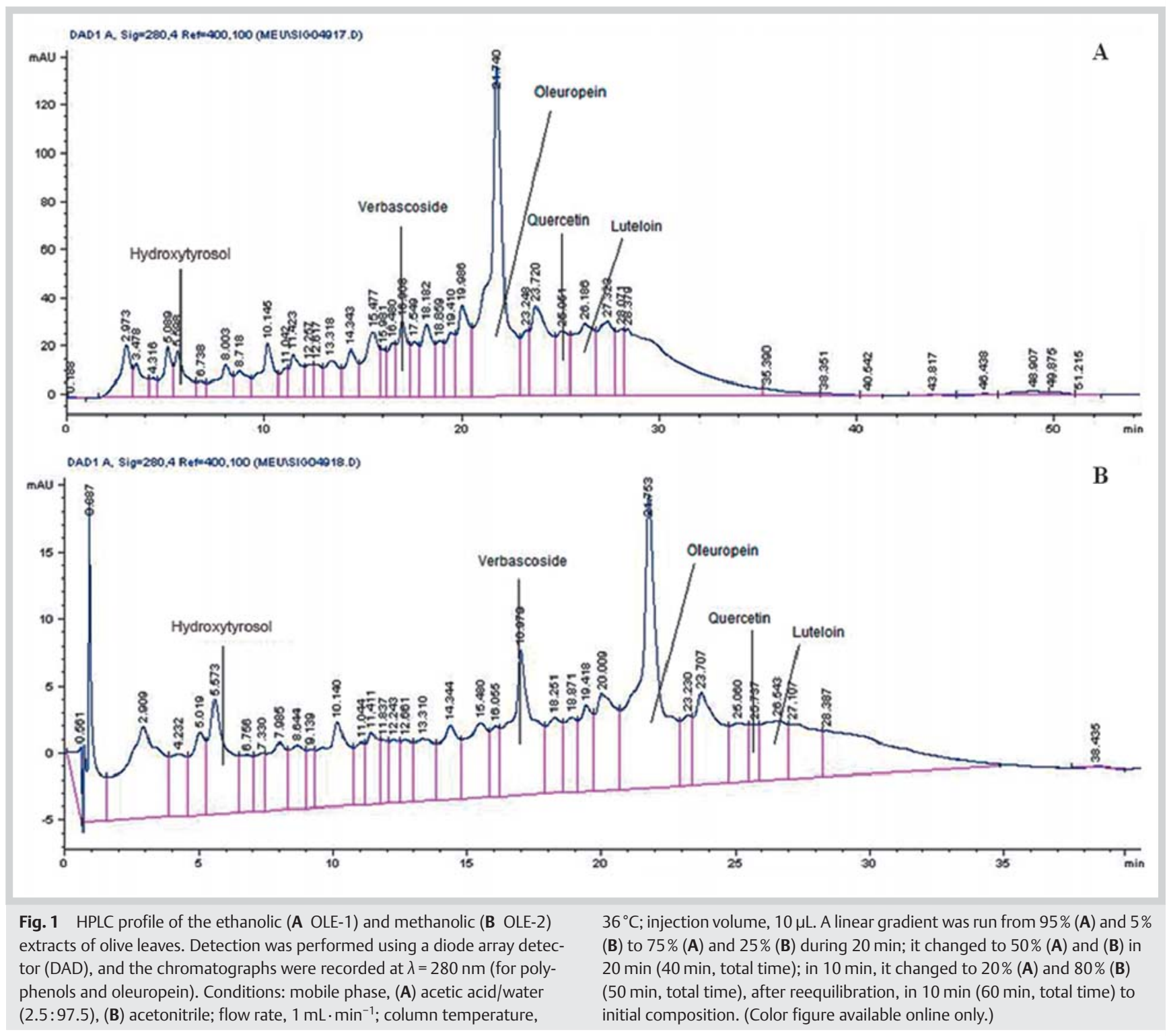

$\left(0.1-10 \mu \mathrm{g} / \mathrm{mL},<\mathrm{IC}_{50}\right)$ of each extract and olive leaf phenolic compounds to test their effects on HNE-induced cell death. MTT findings confirmed that each leaf extract (OLE-1 and OLE-2) has a protective effect on HNE-induced toxicity in H9c2 cells even at a $0.1-\mu \mathrm{g} / \mathrm{mL}$ concentration, which is comparable with the protective effect of oleuropein, hydroxytyrosol, or quercetin ( Fig. 3). OLE-1 has a significantly more protective effect than OLE-2 on the viability of H9c2 cells ( $\bullet$ Fig. 3). The degree of the protective effect of quercetin was similar with the degree of protective effect of OLE-1. In comparison with OLE-1, oleuropein or hydroxytyrosol alone demonstrated a lesser protective effect on the viability in HNE-exposed H9c2 cells (๑ Fig. $\mathbf{3}$ ).

A cell membrane permeable fluorescent dye, DCFH2-DA, that is sensitive to oxidation was used to assess the levels of intracellular oxidative stress after exposure to HNE and also following pretreatment with OLE-1, OLE-2, oleuropein, hydroxytyrosol, and quercetin for $24 \mathrm{~h}$. OLE-2 and oleuropein at 0.1 and $10 \mu \mathrm{g} / \mathrm{mL}$ concentrations, and OLE- 1 and hydroxytyrosol at $10 \mu \mathrm{g} / \mathrm{mL}$ concentrations completely suppressed the ROS production induced by HNE ( Fig. 4). However, OLE-1, hydroxytyrosol, and quercetin at a $0.1 \mu \mathrm{g} / \mathrm{mL}$ concentration were found to have a relatively less

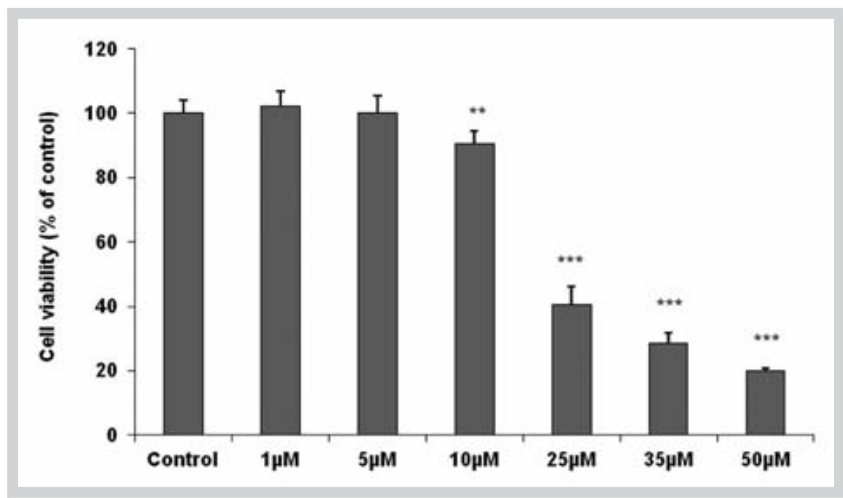

Fig. 2 Cytotoxicity of 4-hydroxynonenal (HNE) in H9c2 (6 h) cells assessed by the MTT viability test. ${ }^{*} \mathrm{P}<0.05,{ }^{* *} \mathrm{p}<0.01,{ }^{* * *} \mathrm{p}<0.001$ vs. control, ANOVA.

suppressive effect than those observed with their $10 \mu \mathrm{g} / \mathrm{mL}$ concentrations against HNE-induced ROS production ( $\odot$ Fig. 4). Ex- 

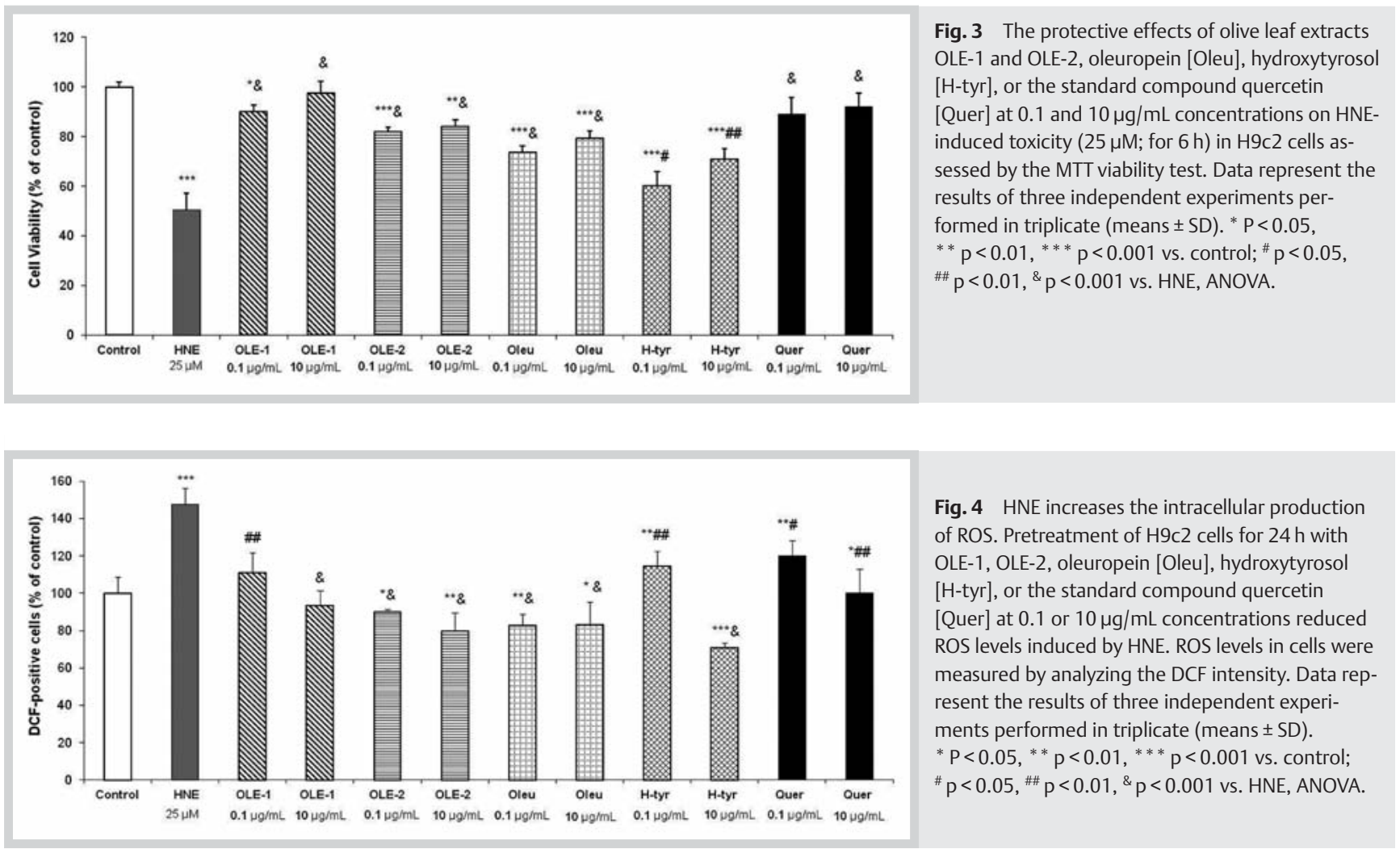

periments showed a predominant ROS scavenging activity at a maximum sample dose of each extract, olive phenolic compound, or standard quercetin $(10 \mu \mathrm{g} / \mathrm{mL})(\mathrm{p}<0.001)$.

Mitochondrial membrane potential was measured by fluorescence staining with JC-1. Stimulation with HNE caused a significant decrease of red fluorescence of JC-1 in H9c2 cells showing mitochondrial dysfunction ( $\bullet$ Fig. $\mathbf{5} \mathbf{a}$ and $\mathbf{b}$ ). Treatment with all preparations significantly prevented the drop of red fluorescence ( $\odot$ Fig. 5 a and b). OLE-1 was found to be less effective than OLE-2 and each olive leaf phenolic compound at $0.1 \mu \mathrm{g} / \mathrm{mL}$ ( $\bullet$ Fig. $\mathbf{5 b}$ ). Western blot analysis of H9c2 cells exposed to HNE revealed expression levels of various proteins, which were partly or markedly downregulated by olive leaf extracts or the components of the extracts represented by commercial oleuropein, hydroxytyrosol, or quercetin as the standard (๑ Fig. 6). HNE exposure to H9c2 cells resulted in significant increases $(p<0.001)$ in stress-activated proteins including p-SAPK/JNK ( Fig. 7a), p-MAPKAPK-2 (๑ Fig. 7b), p-Hsp27 ( Fig. 7c) p-c-Jun, ( Fig. 7d), and clCASP-3 ( Fig. 7e). The HNE-induced increase on p-SAPK/JNK was partly but significantly and concentration-dependently blocked by both extracts and the polyphenolic compounds. At a concentration of $10 \mu \mathrm{g} / \mathrm{mL}$, oleuropein and hydroxytyrosol were found to be more effective than other studied materials to inhibit HNE-induced p-SAPK/JNK ( $\bullet$ Fig. 7 a). In comparison with OLE-1, oleuropein or quercetin and OLE-2 or hydroxytyrosol produced an upwards inhibition on p-MAPKAPK-2 protein levels $(\mathrm{p}<$ 0.001 ) ( Fig. 7 b). In response to carbonyl stress, HSP27 (Ser82) expression increases several folds to create a cellular adaptation to the adverse environmental stimuli. While HSP27 expression was significantly inhibited by all studied materials, OLE-1, oleuropein, and quercetin at a concentration of $10 \mu \mathrm{g} / \mathrm{mL}$ were found to be more effective $(p<0.001)(\diamond$ Fig. $7 c$ ). Olive leaf extracts and the phenolic compounds have an inhibitory effect on c-Jun phosphorylation at their nontoxic concentrations $(p<0.05$ or $\mathrm{p}<0.01$ ), and OLE-2 showed the most significant inhibition on HNE-induced c-Jun phosphorylation at $10 \mu \mathrm{g} / \mathrm{mL}(\mathrm{p}<0.001)$ ( Fig. 7d). The upregulation of cl-CASP3 by HNE was decreased in the presence of phenolic olive leaf constituents. The degrees of attenuations after the treatment of each extract or phenolic phytochemical on HNE-induced cl-CASP3 were found to be similar $(0.1$ or $10 \mu \mathrm{g} / \mathrm{mL}, \mathrm{p}<0.05$ or $\mathrm{p}<0.01)$ and smaller than the inhibitory effect of quercetin ( $p<0.001$ ) ( $\odot$ Fig. 7 e).

\section{Discussion}

$\nabla$

In this study, an HNE-induced toxicity model was used to investigate the effects of carbonyl compound-induced redox stress and the protective ability of olive leaf phenolic compounds and extracts on cardiomyocyte viability and the apoptotic process in H9c2 cells in vitro. We found that (1) a carbonyl compound, HNE, inhibits viability and accelerates apoptosis in a concentration-dependent manner and (2) OLE-1, OLE-2 and each phenolic component of olive leaves, like oleuropein, hydroxytyrosol, and quercetin, exert a significant antiapoptotic effects against HNEinduced toxicity in $\mathrm{H} 9 \mathrm{c} 2$ cells. The protective action mechanisms of the extracts or the phenolic compounds were accompanied by a decrease of the induction of the apoptotic stress markers ( $p$ MAPKAPK-2, p-SAPK/JNK, p-HSP27, p-c-JUN, and cl-CASP3). The findings were related to the cellular protective effects of the extracts, and oleuropein and hydroxytyrosol were comparable with the effects of positive standard $[21,22]$. The protective action mechanisms of the extracts and the phenolic constituents of olive leaves include the reduction of ROS production and the amelioration of the mitochondria membrane potential in H9c2 cells. These results suggest that the ethanolic extract of olive leaves and also its polyphenolic constituents have a key role in cardiomyocyte protection. 

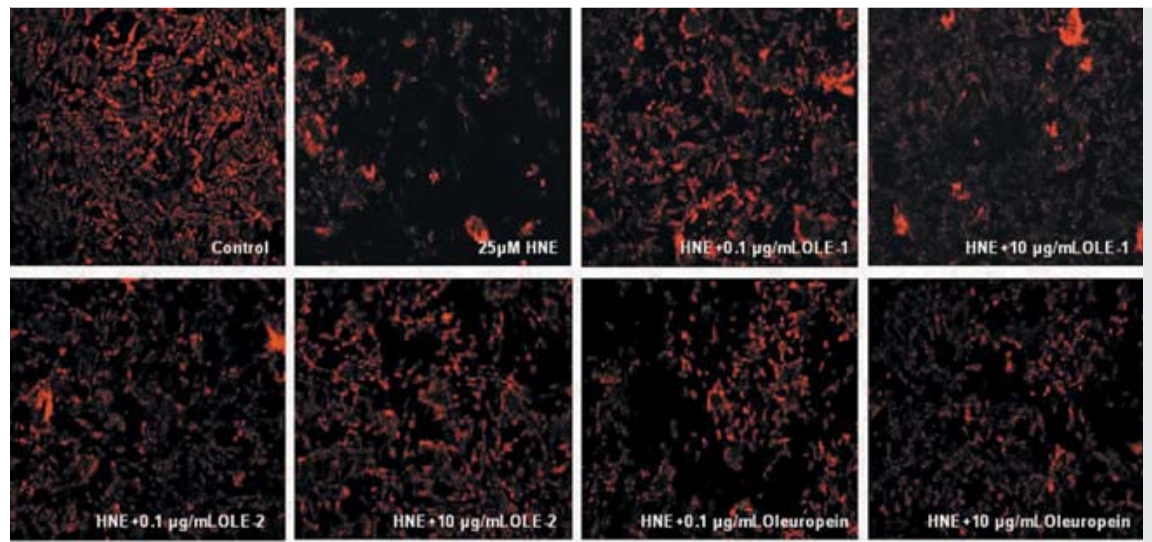

Fig. 5a JC-1 staining for 4-hydroxynonenal-induced dissipation of mitochondrial membrane potential in $\mathrm{H} 9 \mathrm{c} 2$ cells. The cells were treated with $0.1 \mu \mathrm{g} / \mathrm{mL}$ and $10 \mu \mathrm{g} / \mathrm{mL}$ doses of OLE-1, OLE-2, oleuropein [Oleu], hydroxytyrosol [H-tyr], or the standard compound quercetin [Quer] for 15 minutes and stained with JC-1, a mitochondrial potential sensitive dye. a Fluorescence pattern of different treatment groups as indicated in the pictures appearing as an increment of red JC-1 aggregates; decreasing green fluorescence means the diminution of mitochondrial depolarization decreases.

(Color figure available online only.)
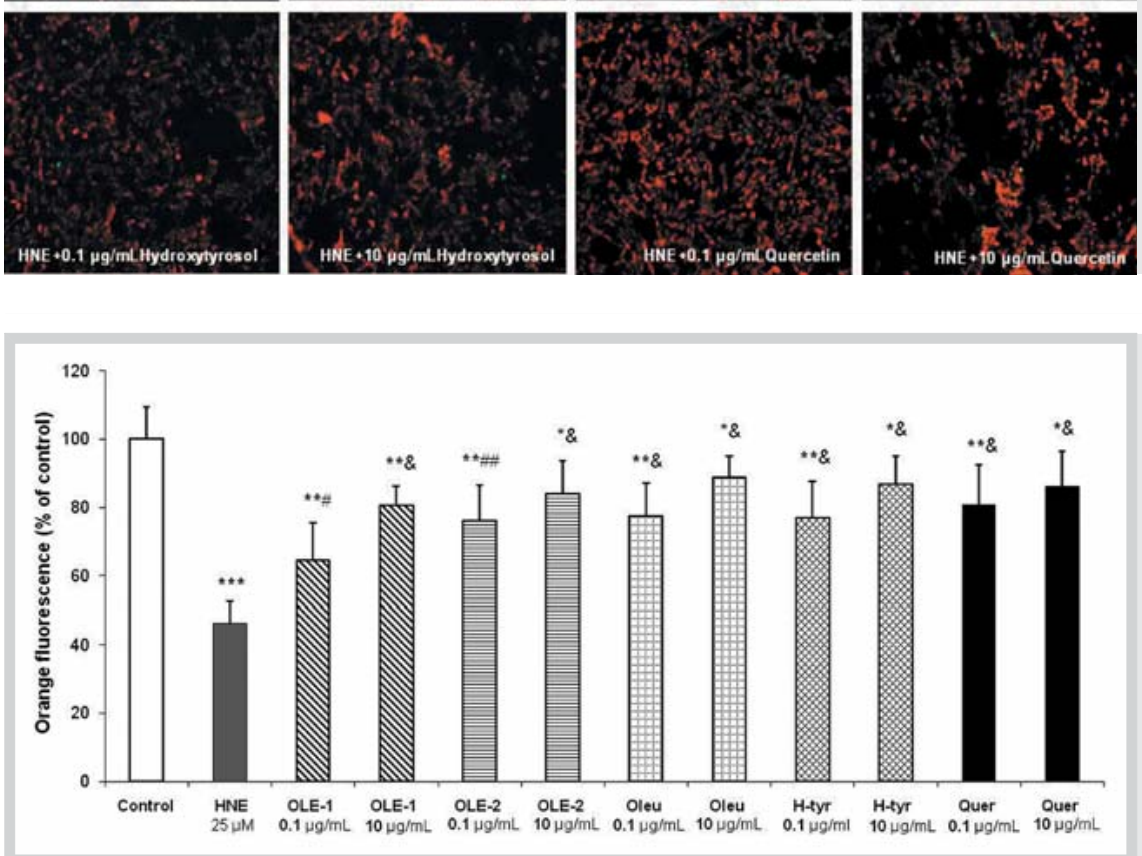

Fig. 5b Quantitative presentation of the data as (mean \pm SD of three samples) percent of positive JC1 stained cells. ${ }^{*} \mathrm{P}<0.05,{ }^{* *} \mathrm{p}<0.01,{ }^{* * *} \mathrm{p}<0.001$ vs. control; ${ }^{\#} p<0.05,{ }^{\# \#} p<0.01,{ }^{\&} p<0.001$ vs. HNE, ANOVA.

HNE, a highly reactive $\alpha, \beta$-aldehydic product generated from polyunsaturated fatty acid peroxidation, is able to accumulate in the heart in response to chronic oxidant infestation [23]. Longterm HNE exposure leads to an increase in HNE-protein adduct formation and dysfunctional proteins, mediating molecular mechanism of carbonyl stress-induced cardiotoxicity [24]. Although carbonyl-induced redox stress is known to aggravate cardiac abnormalities $[7,8]$, there is no report showing stress-induced signaling pathways after HNE exposure in vitro in cardiomyocte cell lines except for one, which reported a link with increased endoplasmic reticulum stress [25].

Upon stimulation by carbonyl stress, p38MAPK is phosphorylated. It then phosphorylates MAPKAPK2 to phosphorylate and activate HSP27 $[26,27]$. The phosphorylation of HSP27 is under the control of the intracellular ROS level [28]. We observed that HSP27 is phosphorylated during HNE exposure in H9c2; on the other hand, the pretreatment with olive leaf phenolic compounds led to a decrease in this phosphorylation of HSP27. This indicates that olive leaf phenolic compounds contribute to H9c2 cell survival via decreasing lethal effects of HNE-induced ROS, which eliminates the requirement for HSP27-regulated prosurvival pathways. Quercetin has been shown to restrict the adaptation-induced MAPKAPK2 and HSP70 synthesis to HNE. The blockade of HSP27 synthesis by quercetin was demonstrated previously by authors in H9c2 cells [29].
c-Jun N-terminal kinase-1 (JNK-1) is activated by oxidative stress as a consequence of HNE induction [30] and thought to promote injury during severe acute myocardial infarction [26]. Several studies reported that JNK is another protein activated by oxidative stress, which is a consequence of HNE induction [31]. The present study demonstrated, for the first time, that JNK is a downstream signaling molecule in HNE-mediated cardiotoxicity in $\mathrm{H} 9 \mathrm{c} 2$ cells. HNE-induced $\mathrm{p}-\mathrm{SAPK} / \mathrm{JNK}$ was downregulated partly by the extracts oleuropein, hydroxytyrosol, or quercetin as concentration-dependently. The results indicated that HNE induction in c-Jun, an important transcription factor that is activated by JNK, was attenuated by olive leaf extracts, especially by treatment with OLE-2 and hydroxytyrosol. Quercetin, which has been shown to be an inhibitor of c-Jun N-terminal kinase in ischemia/reperfusion injury in isolated rat hearts [32], attenuated HNE-induced c-Jun in H9c2 cells. Quercetin is the most abundant flavonoid [21] and is believed to attenuate cardiovascular disease and inhibit cardiac hypertrophy by blocking c-Jun [33].

Another important intracellular signaling pathway leading to ROS-mediated apoptosis is involved in the activation of the caspases. Activation of p38 MAPK then leads to cleavage of proCASP3, yielding active cleaved-CASP3, one of the key effectors of apoptosis [34]. It has been reported that, upon oxidant exposure, H9c2 cells exhibit increased activation of CASP3, which is considered an indicator of cell death [35]. In our study, oleuropein, hy- 


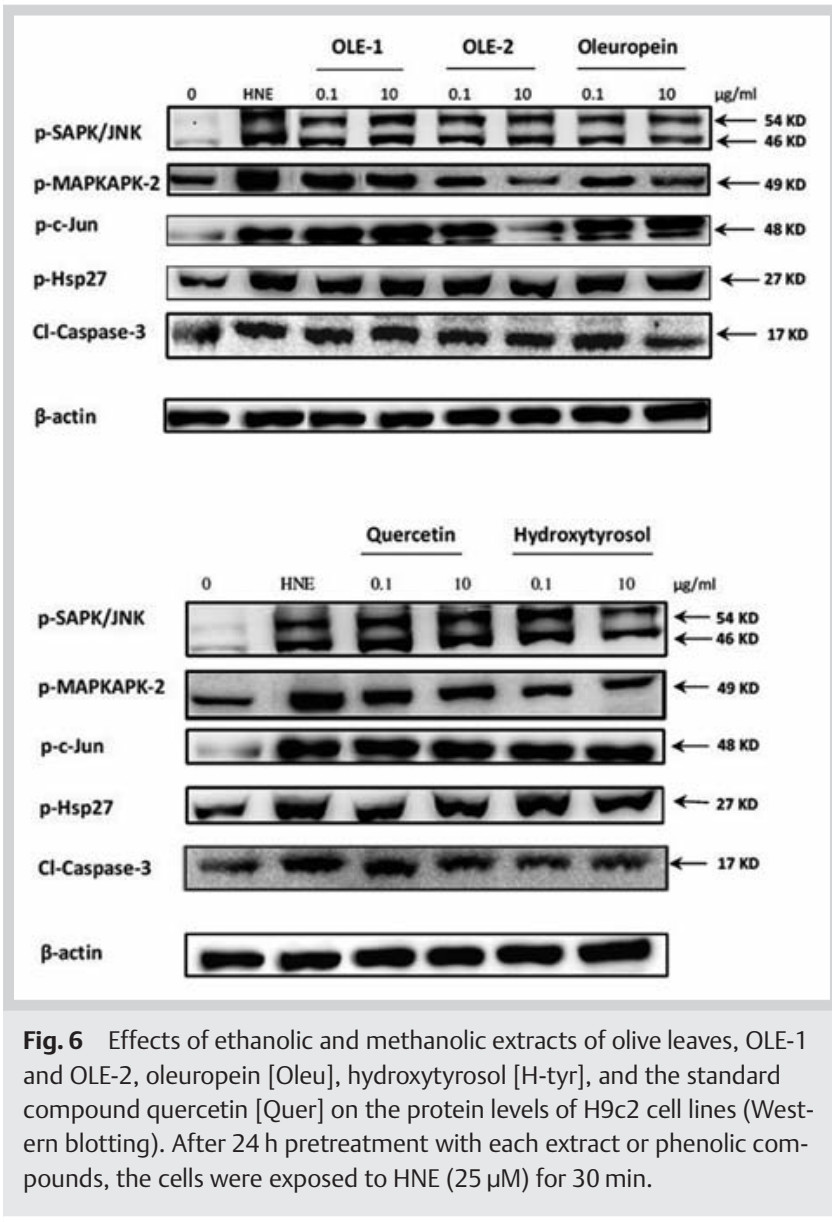

droxytyrosol, and both extracts reduced CASP3 activity by modulating the mitochondrial response to HNE. It has been demonstrated that quercetin exerts protective effects against $\mathrm{H}_{2} \mathrm{O}_{2}$ cardiotoxicity as confirmed by measurement of caspase- 3 activity and that the mechanism of its action involved in the modulation of PI3K/Akt and ERK1/2 signaling pathways in H9c2 cardiomyoblasts [36]. Overall, the effects of different olive leaf extracts on apoptotic markers were different: OLE-1 exerted an inhibitory effect more than OLE-2 on HNE-induced p-SAPK/JNK and pHsp27, while p-MAPKAPK-2 and p-c-Jun were more downregulated by OLE-2 than OLE-1.

The cardiomyocyte protection observed in the present study by olive leaf extracts or their phenolic components against HNE toxicity are consistent with our previous findings showing a powerful protection of the ethanolic extract from olive leaves ( 20\% oleuropein content) in insulin-releasing cells against $\mathrm{H}_{2} \mathrm{O}_{2}$-induced [19] or cytokine-induced [20] toxicity and apoptosis. Based on the present findings, we suggest a model representing the action mechanisms of olive leaf phenolic compounds on HNE-induced apoptotic cell death and toxicity ( $\bullet$ Fig. 8).

The present study established that OLE-1 has more ability than OLE2 or each phenolic compound to protect cardiomyocytes against HNE-induced stress and toxicity. The more effective protecting ability of OLE-1 on cardiomyocyte viability is likely due to the synergistic interactions of polyphenolics and other compounds of OLE- 1 since OLE- 1 also includes a larger amount of other phenolics (verbascosid and luteolin, as well as oleuropein, hydroxytyrosol, and quercetin) than OLE-2. In this respect, we highlight once more that the usage of a polyphenolic extract mixture can be preferred for cell protection in comparison with a
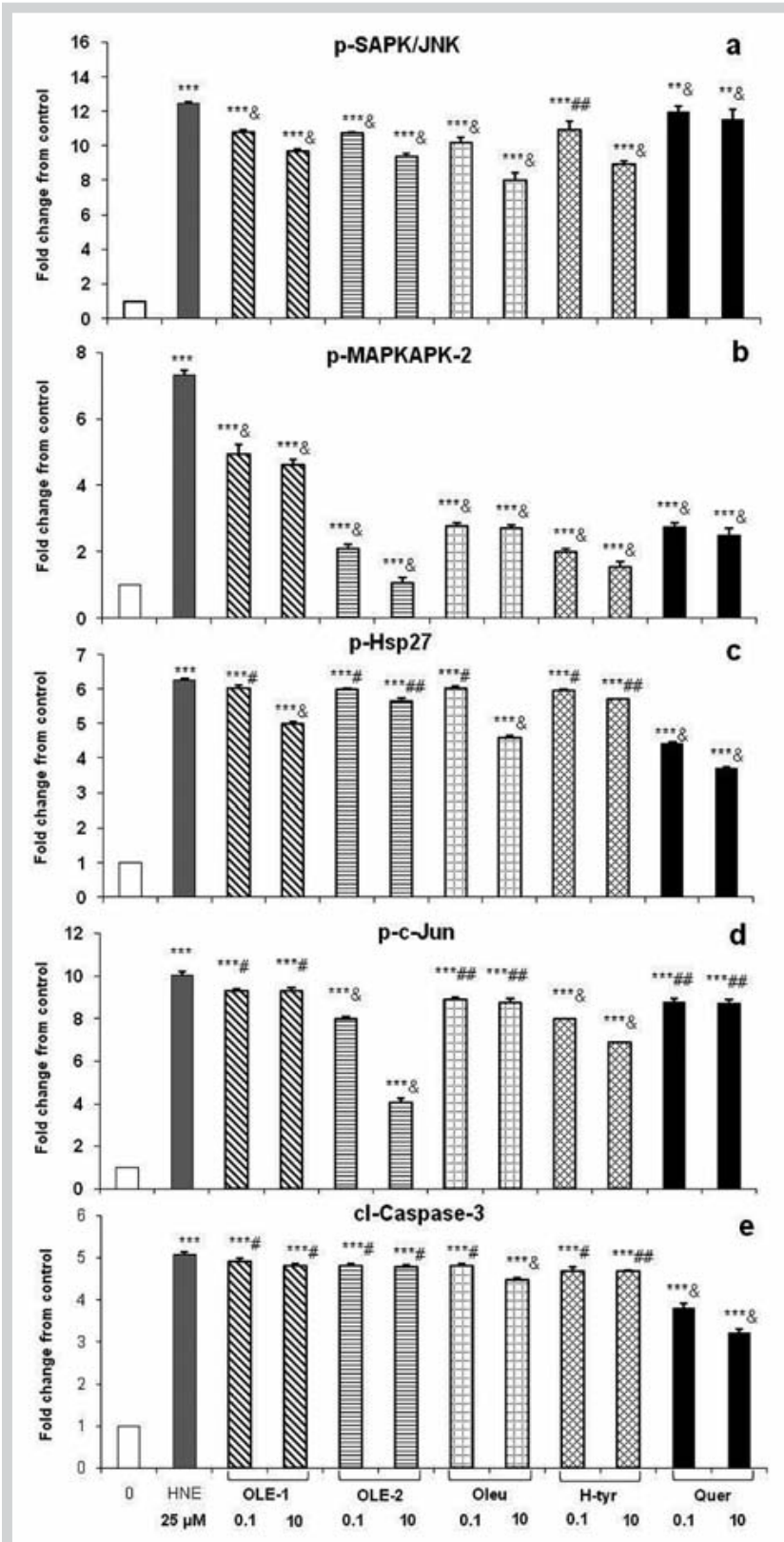

Fig. 7 Western blot analysis demonstrated that the expressions of some different stress-stimulated and apoptotic signaling proteins ( $\mathrm{p}-\mathrm{SAPK} / \mathrm{JNK}$, p-MAPKAPK-2, p-c-Jun, p-Hsp27, cl-Caspase-3) significantly increased after $25 \mu \mathrm{M}$ 4-hydroxynonenal treatment of H9c2 cells. However, OLE-1, OLE-2, oleuropein [Oleu], hydroxytyrosol [H-tyr], or quercetin [Quer] $(0.1$ or $10 \mu \mathrm{g} / \mathrm{mL}$ ) significantly decreased HNE-induced protein expression. The protein bands of p-SAPK/JNK, p-MAPKAPK-2, p-c-Jun, p-Hsp27, and ClCaspase-3 were obtained from Western blot analysis. They were from a representative experiment repeated three times with similar results. The expression level of the proteins was quantitated by densitometric analysis by using Image I Software Informer, and the mean densitometry values from three independent Western blot experiments are expressed relative to respective untreated controls (relative optical density $=1$ ). Oleu: oleuropein; H-tyr: hydroxytyrosol; ${ }^{*} p<0.05,{ }^{* *} p<0.01,{ }^{* * *} p<0.001$ vs. control; ${ }^{p} p<0.05,{ }^{\# \#} p<0.01,{ }^{\&} p<0.001$ vs. HNE, ANOVA.

mono-component antioxidant compound [24]. The leaves of the olive plant (0. europaea) have been commonly used for centuries in folk medicine. Recently, the medicinal properties of olive products have focused on its polyphenols (particularly oleuropein and hydroxytyrosol), which, according to animal and in vitro studies, 


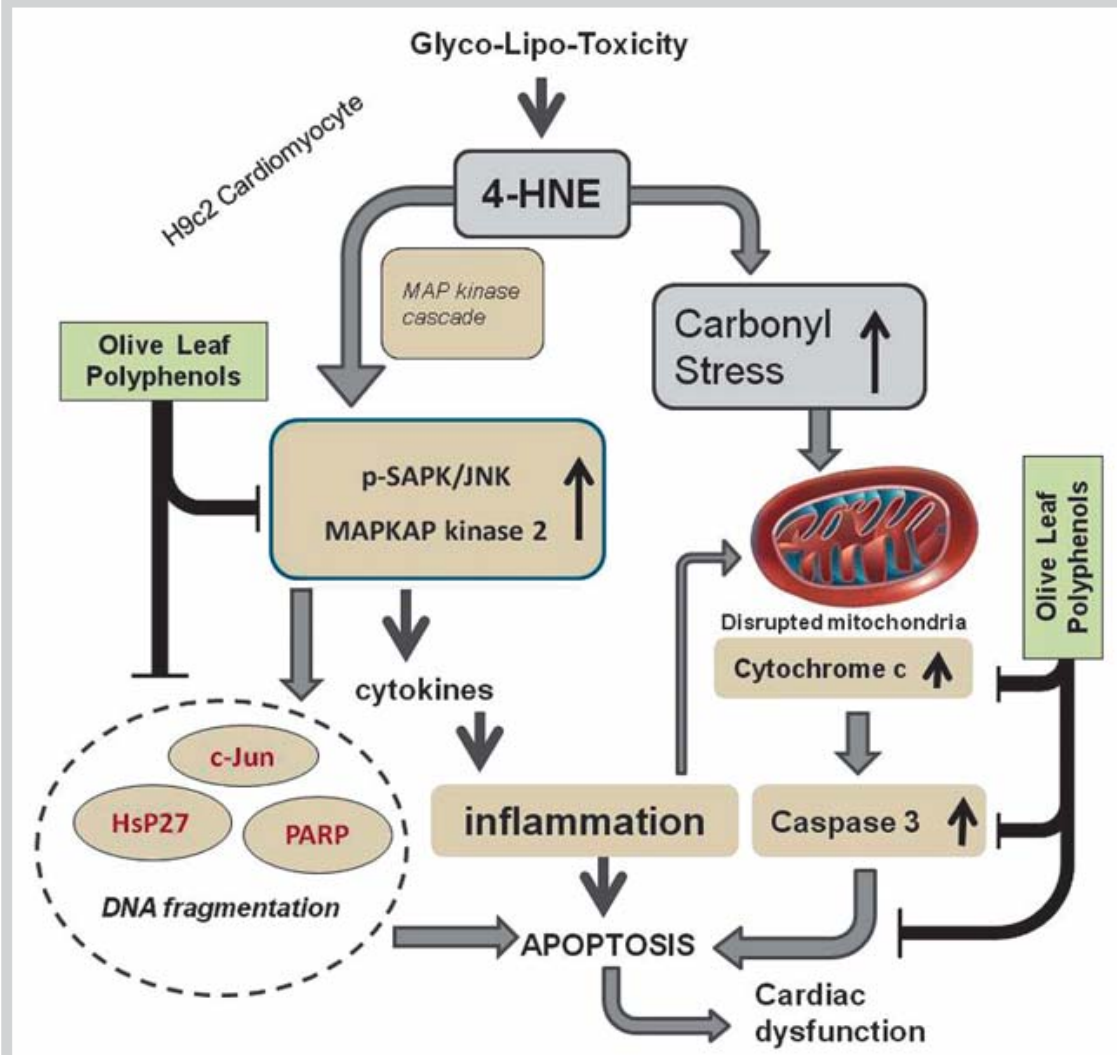

Fig. 8 Intracellular action mechanisms of olive leaf extracts and phenolic compounds exerting protective effects on cardiomyocytes following 4-hydroxynonenal exposure-induced carbonyl stress and toxicity. (Color figure available online only.)

have antioxidant, hypoglycemic, antihypertensive, and antiatherosclerotic properties, and improve metabolic syndrome and cardiovascular risk factors in patients [37].

In conclusion, we provided the first evidence for cardiomyocyte protection by olive leaf phenolic extracts and the major phenolic components oleuropein and hydroxytyrosol against HNE-induced cytotoxicity. This study supports the results of animal or clinical studies demonstrating the cardioprotective effects of the olive leaf and its phenolic constituents along with elucidating their cellular action mechanisms and effects on downstream stress signaling pathways.

\section{Materials and Methods \\ $\nabla$}

\section{Chemicals}

Folin-Ciocalteau reagent and gallic acid were from Merck Co. All cell culture products were obtained from Lonza. HNE was purchased from Cayman Chemical. Oleuropein (HPLC grade $\geq 96 \%$; Applichem A6909 Lot\# 12101123) and hydroxytyrosol (HPLC grade > 98\%; Applichem A5303 Lot\# 11120406) were purchased from Applichem. Quercetin (HPLC grade $\geq 98 \%$ ) was from SigmaAldrich (Q4951 Lot\# 060M1196 V).

\section{Extracts}

The ethanolic and methanolic extracts of olive leaves prepared according to a modified method by the authors $[12,13]$ were obtained from Farmasens Co. as a gift. OLE-1 (Lot\# 012-08) and OLE-2 (Lot\# 005-10) were stored in light-protected glass bottles at $4{ }^{\circ} \mathrm{C}$ until further analyzed.

\section{Determination of the total phenolic content}

Total phenolic content was determined using the Folin-Ciocalteau assay [14], which is based on colorimetric oxidation and reduction reaction. An aliquot $(0.5 \mathrm{~mL})$ of each extract was reacted with freshly prepared $1.25 \mathrm{~mL}$ of $20 \%$ sodium carbonate and $0.5 \mathrm{~mL}$ of $1 \mathrm{~N}$ Folin reagent in a screw-capped test tube. Required dilutions were prepared with distilled water. Test tubes were vortexed and after $40 \mathrm{~min}$, absorbance readings were recorded at $725 \mathrm{~nm}$ by a UV-Vis spectrophotometer (Shimadzu). The amount of total phenolic compounds was expressed as milligram of gallic acid equivalent per gram of dry weight of sample.

\section{High-performance liquid chromatography determination}

The modified HPLC analysis given in the literature $[13,14]$ was used for the quantification of phenolic compounds of the ethanolic and methanolic extracts of olive leaves. The HPLC equipment used was a Hewlett-Packard Series HP 1100 equipped with a diode array detector, and the chromatographs were recorded at $\lambda=260 \mathrm{~nm}$ (for hydroxytyrosol and oleuropein). The stationary phase was a $\mathrm{C}_{18}$ LiChrospher 100 analytical column $(250 \times 4 \mathrm{~mm}$ i.d.) with a particle size of $5 \mu \mathrm{m}$ (Merck) thermostated at $30^{\circ} \mathrm{C}$. The HPLC chromatogram of the olive leaf extract was compared to the standard mix of identified compounds. The compounds in the olive leaf extracts were identified by comparison of their retention times with the corresponding standards.

\section{Cell culture}

The embryonic rat heart-derived myogenic cell line H9c2 was purchased from the American Tissue Type Collection (\#CRL1446). The H9c2 cells were cultured in $75-\mathrm{cm}^{2} \mathrm{TC}$ flasks in DMEM supplemented with $10 \%$ FBS, 2 mM L-glutamine, and 1\% penicil- 
lin-streptomycin. The cells were cultured at $37^{\circ} \mathrm{C}$ in a humidified atmosphere containing $95 \%$ air $/ 5 \% \mathrm{CO}_{2}$.

\section{Application of the extracts and phenolic compounds}

Stock solutions of olive leaf extracts were dissolved in 10\% DMSO, and the concentrations of the extracts were diluted with DMEM. The extracts at the maximum concentration contained $0.02 \%$ DMSO. Hydroxytyrosol, oleuropein, and quercetin were dissolved in media.

\section{Cell viability assay}

The effect of each extract or phenolic compound on the viability of H9c2 cells in the presence of HNE was detected by the MTT method [38]. The cells $\left(1 \times 10^{4} /\right.$ well $)$ were seeded in 96 -well plates and incubated for $24 \mathrm{~h}$. Later, fresh medium containing $0.1,10,25$, and $50 \mu \mathrm{g} / \mathrm{mL}$ concentrations of each extract or phenolic compound was added and the cells were incubated for $24 \mathrm{~h}$ followed by incubation with $\operatorname{HNE}(25 \mu \mathrm{M})$ for $6 \mathrm{~h}$. Then $10 \mu \mathrm{L}$ of MTT stock solution $(5 \mathrm{mg} / \mathrm{mL}$ ) was added, and the plates were incubated at $37^{\circ} \mathrm{C}$ for $4 \mathrm{~h}$. Culture medium was removed and the resultant formazan crystals were dissolved in $100 \mu \mathrm{L}$ DMSO, and the absorbance values were read on a microplate reader (PowerWave XS2; BioTek Instruments) at the wavelength of $572 \mathrm{~nm}$. The cells were assayed in quadruplicate, and at least two independent experiments were carried out.

\section{Measurement of reactive oxygen species}

The accumulation of intracellular ROS was determined by measuring DCF fluorescence and evaluated with a modified method described by [39]. ROS causes oxidation of DCFH2-DA to the fluorescent product DCF in the cell. In brief, the cells $\left(2 \times 10^{4} /\right.$ well $)$ were pretreated with each OLE or the phenolic compounds in doses of 0.1 and $10 \mu \mathrm{g} / \mathrm{mL}$ and then exposed to HNE for $15 \mathrm{~min}$ at $37^{\circ} \mathrm{C}$ in darkness. Cell culture plates were washed and incubated with $10 \mu \mathrm{M}$ DCFH2-DA (Molecular Probes) in medium. The DCFH2-DA-containing medium was removed; the cells were washed twice with PBS, and DCF fluorescence was quantified (Ex $485 \mathrm{~nm} / \mathrm{Em} 535 \mathrm{~nm}$ ) using a multimode microplate reader (PowerWave XS2; BioTek Instruments).

\section{Determination of mitochondrial membrane potential}

$\Delta \Psi$ m was indirectly determined using a JC-1 assay kit (Cayman Chemical Company). JC-1, a fluorescent dye, and the lipophilic cationic probe 5,5',6,6'-tetrachloro-1,l',3,3'-tetraethyl benzimidazol carbocyanine iodide were used for investigating $\Delta \Psi \mathrm{m}$ in living cells. An unincorporated dye exists in a monomeric form, emitting at $527 \mathrm{~nm}$ after excitation at $490 \mathrm{~nm}$ [40]. To assess the integrity of $\Delta \Psi \mathrm{m}$, the cells were pretreated with each extract or phenolic compounds in 0.1 and $10 \mu \mathrm{g} / \mathrm{mL}$ concentrations for $24 \mathrm{~h}$. After pretreatment, the cells were incubated with $25 \mu \mathrm{M}$ HNE for $15 \mathrm{~min}$ and then stained with $10 \mu \mathrm{M} \mathrm{JC}-1$ in medium at $37^{\circ} \mathrm{C}$ for $30 \mathrm{~min}$ in darkness. Then cells were washed twice with PBS. The JC-1 fluorescence was measured by fluorescence microscopy or spectrofluorometry. Fluorescence images were captured and processed using a Leica DM6000 microscope with a Leica DFC420 camera controlled via Leica LAS AF7000 software. Fluorescence signals were also measured by spectrofluorometry in $485 \mathrm{~nm}$ excitation, $525 \mathrm{~nm}$, and $590 \mathrm{~nm}$ for emission of green and red fluorescence, respectively. The ratio of red/green fluorescence of JC- 1 was used as an indicator of mitochondrial function. In healthy cells with high mitochondrial $\Delta \Psi \mathrm{m}$, JC- 1 forms multimers called J-aggregates that produce red fluorescence. On the other hand, in apoptotic or unhealthy cells with low $\Delta \Psi \mathrm{m}$, JC-1 remains in the monomeric form, which shows green fluorescence. The results are illustrated as the percentage change in fluorescence values in comparison with the untreated controls.

\section{Western blotting}

H9c2 cells cultured in $60-\mathrm{mm}$ petri dishes (Sarstedt) were lysed in $250 \mu \mathrm{L}$ of lysis buffer (1\% Triton, $20 \mathrm{mM}$ Tris- $\mathrm{HCl}$ [pH 7.5], $150 \mathrm{mM} \mathrm{NaCl}, 1 \mathrm{mM} \mathrm{Na}{ }_{2}$ EDTA, 1 mM EGTA, 2.5 mM sodium pyrophosphate, $1 \mathrm{mM}$ beta-glycerophosphate, $1 \mathrm{mM} \mathrm{Na}_{3} \mathrm{VO}_{4}$, and $1 \mu \mathrm{g} / \mathrm{mL}$ leupeptin) supplemented with $1 \mathrm{mM}$ PMSF (Roche Diagnostics). Protein concentrations were determined by using the BCA protein assay (Pierce). Protein lysates $(20 \mu \mathrm{g})$ were heated for 5 min at $94^{\circ} \mathrm{C}$ in Laemmli sample buffer containing $5 \% \beta$-mercaptoethanol and loaded on 4-15\% Tris-glycine SDS-PAGE gels, then transferred electrophoretically to nitrocellulose membranes. Membranes were blocked with $5 \%$ nonfat dry milk for $1 \mathrm{~h}$ and incubated overnight at $4{ }^{\circ} \mathrm{C}$ with phospho-MAPKAPK-2 (Thr334), phospho-HSP27 (Ser82), phospho-SAPK/JNK (Thr183/ Tyr185), phospho-c-Jun (Ser73), and cleaved Caspase-3 (Asp175) antibodies (Cell Signaling Technology). Protein bands were detected with horseradish peroxidase-conjugated anti-rabbit secondary antibodies (Cell Signaling Technology) and visualized by West-Femto ECL reagents (Pierce). Chemiluminescent signals of immunoblots were documented by using Gel Logic 2200 Pro (Carestream Health). The intensity of specific proteins was quantified by using Carestream Molecular Image Software and ImageJ Software (v. 1.4; National Institutes of Health).

\section{Statistical analysis}

Experiments were performed at least three times (more than three times for MTT and DCF experiments), and statistical analysis was performed with the unpaired Student's t-test using XLPlot v. 2.81. One-way ANOVA tests were performed using program Past, v. 2.16. All values are expressed as means $\pm S D$, and $\mathrm{p}<0.05, \mathrm{p}<0.01$, and $\mathrm{p}<0.001$ were considered statistically significant.

\section{Acknowledgements \\ $\nabla$}

This work was supported by Gazi University-BAP (01/2012-70), EU-COST-BM1203 (EU-ROS), and KOSGEB (2011-0850). We also thank Farmasens Co., Gazi University Techno Park, Ankara, Turkey, (www.farmasens.com) for their support to Bio. E. Burcu Bali as a researcher.

The preliminary findings of this study have been presented in (1) the "First Mediterranean Symposium on Aromatic and Medicinal Plants", April 17-20, 2013, North Cyprus, http://www.mesmap2013.emu.edu.tr: Karasu C. Cellular redox regulation by olive and pomegranate extracts accompany to cardioprotection and neuroprotection; and (2) "MMC-2012, International Conference on Molecular Medicine: Alternative Strategies against Cancer \& Inflammation", December 19-22, 2012 Bangkok; http://www. mmc2012.org": Karasu C. The protective ability of the Anatolian plant extracts against glycoxidation and oxidative protein damage.

\section{Conflict of Interest \\ $\nabla$}

The authors declare no conflict of interest. 
Affiliations

${ }^{1}$ Cellular Stress Response \& Signal Transduction Research Laboratory, Department of Medical Pharmacology, Faculty of Medicine, Gazi University, and Farmasens Biotech Co., Gazi TechnoPark, Ankara, Turkey

2 Department of Medical Biology \& Genetics, Faculty of Medicine, Gazi University, Ankara, Turkey

${ }^{3}$ Institute of Experimental Pharmacology and Toxicology, Slovak Academy of Sciences, Bratislava, Slovak Republic

${ }^{4}$ Department of Chemical \& Biochemical Engineering, Izmir Institute of Technology, and DUAG Co., IYTE, İzmir, Turkey

5 Department of Pharmacognosy, Faculty of Pharmacy, Gazi University, Ankara, Turkey

\section{References}

1 Poli G, Schaur RJ, Siems WG, Leonarduzzi G. 4-hydroxynonenal: a membrane lipid oxidation product of medicinal interest. Med Res Rev 2008; 28: 569-631

2 Uchida K, Stadtman ER. Modification of histidine residues in proteins by reaction with 4-hydroxynonenal. Proc Natl Acad Sci U S A 1992; 89: 4544-4548

3 Szapacs ME, Riggins JN, Zimmerman LJ, Liebler DC. Covalent adduction of human serum albumin by 4-hydroxy-2-nonenal: kinetic analysis of competing alkylation reactions. Biochemistry 2006; 45: 10521-10528

4 Grune T, Davies KJ. The proteasomal system and HNE-modified proteins. Mol Aspects Med 2003; 24: 195-204

5 Galvani S, Coatrieux C, Elbaz M, Grazide MH, Thiers JC, Parini A, Uchida K, Kamar N, Rostaing L, Baltas M, Salvayre R, Nègre-Salvayre A. Carbonyl scavenger and antiatherogenic effects of hydrazine derivatives. Free Radic Biol Med 2008; 45: 1457-1467

6 Miller DM, Singh IN, Wang JA, Hall ED. Administration of the Nrf2-ARE activators sulforaphane and carnosic acid attenuates 4-hydroxy-2nonenal-induced mitochondrial dysfunction ex vivo. Free Radic Biol Med 2013; 57: 1-9

7 Karasu C. Glycoxidative stress and cardiovascular complications in experimentally-induced diabetes: effects of antioxidant treatment. Open Cardiovasc Med J 2010; 4: 240-256

8 Sakul A, Cumaoğlu A, Aydin E, Ari N, Dilsiz N, Karasu C. Age- and diabetes-induced regulation of oxidative protein modification in rat brain and peripheral tissues: consequences of treatment with antioxidant pyridoindole. Exp Gerontol 2013; 48: 476-484

9 Qin F, Simeone M, Patel R. Inhibition of NADPH oxidase reduces myocardial oxidative stress and apoptosis and improves cardiac function in heart failure after myocardial infarction. Free Radic Biol Med 2007; 43: 271-281

10 Anderson EJ, Katunga LA, Willis MS. Mitochondria as a source and target of lipid peroxidation products in healthy and diseased heart. Clin Exp Pharmacol Physiol 2012; 39: 179-193

11 He L, Liu B, Dai Z, Zhang HF, Zhang YS, Luo XJ, Ma QL, Peng J. Alpha lipoic acid protects heart against myocardial ischemia-reperfusion injury through a mechanism involving aldehyde dehydrogenase 2 activation. Eur J Pharmacol 2012; 678: 32-38

12 Bayçin D, Altiok E, Ulkü S, Bayraktar O. Adsorption of olive leaf (Olea europaea L.) antioxidants on silk fibroin. J Agric Food Chem 2007; 55 : 1227-1236

13 Altıok E, Baycin D, Bayraktar O, Semra Ulkü S. Isolation of polyphenols from the extracts of olive leaves (Olea europaea L.) by adsorption on silk fibroin. Sep Purif Technol 2008; 62: 342-348

14 Dekanski D, Janicijevic-Hudomal S, Tadic V, Markovic G, Arsic I, Mitrovic $D M$. Phytochemical analysis and gastroprotective activity of an olive leaf extract. J Serb Chem Soc 2009; 74: 367-377

15 Mylonaki S, Kiassos E, Makris DP, Kefalas P. Optimisation of the extraction of olive (Olea europaea) leaf phenolics using water/ethanol-based solvent systems and response surface methodology. Anal Bioanal Chem 2008; 392: 977-985

16 Koca U, Süntar I, Akkol EK, Yilmazer D, Alper M. Wound repair potential of Olea europaea L. leaf extracts revealed by in vivo experimental models and comparative evaluation of the extracts' antioxidant activity. J Med Food 2011; 14: 140-146

17 Poudyal H, Campbell F, Brown L. Olive leaf extract attenuates cardiac, hepatic, and metabolic changes in high carbohydrate-, high fat-fed rats. J Nutr 2010; 140: 946-953

18 Manna C, Migliardi V, Golino P, Scognamiglio A, Galletti P, Chiariello M, Zappia $V$. Oleuropein prevents oxidative myocardial injury induced by ischemia and reperfusion. J Nutr Biochem 2004; 15: 461-466

19 Cumaoğlu A, Rackova L, Stefek M, Kartal M, Maechler P, Karasu C. Effects of olive leaf polyphenols against $\mathrm{H}_{2} \mathrm{O}_{2}$ toxicity in insulin secreting $\beta$ cells. Acta Biochim Pol 2011; 58: 45-50
20 Cumaoğlu A, Ari N, Kartal M, Karasu C. Polyphenolic extracts from Olea europaea L. protect against cytokine-induced $\beta$-cell damage through maintenance of redox homeostasis. Rejuvenation Res 2011; 14: 325334

21 Stefek $M$, Karasu C. Eye lens in aging and diabetes: effect of quercetin. Rejuvenation Res 2011; 14: 525-534

22 Hur SJ, Lee SJ, Kim DH, Chun SC, Lee SK. Onion extract structural changes during in vitro digestion and its potential antioxidant effect on brain lipids obtained from low- and high-fat-fed mice. Free Radic Res 2013; 47: 1009-1015

23 Budas GR, Disatnik MH, Chen CH, Mochly-Rosen D. Activation of aldehyde dehydrogenase 2 (ALDH2) confers cardioprotection in protein kinase C epsilon (PKCvarepsilon) knockout mice. J Mol Cell Cardiol 2010; 48: 757-764

24 Ergin V, Hariry RE, Karasu C. Carbonyl stress in aging process: role of vitamins and phytochemicals as redox regulators. Aging Dis 2013; 4: 276-294

25 Liao J, Sun A, Xie Y, Isse T, Kawamoto T, Zou Y, Ge J. Aldehyde dehydrogenase-2 deficiency aggravates cardiac dysfunction elicited by endoplasmic reticulum stress induction. Mol Med 2012; 18: 785-793

26 Wei J, Wang W, Chopra I, Li HF, Dougherty CJ, Adi J, Adi N, Wang H, Webster KA. c-Jun N-terminal kinase (JNK-1) confers protection against brief but not extended ischemia during acute myocardial infarction. J Biol Chem 2011; 286: 13 995-14006

27 Rouse J, Cohen P, Trigon S, Morange M, Alonso-Llamazares A, Zamanillo $D$, Hunt T, Nebreda AR. A novel kinase cascade triggered by stress and heat shock that stimulates MAPKAP kinase-2 and phosphorylation of the small heat shock proteins. Cell 1994; 78: 1027-1037

28 Mehlen P, Arrigo AP. The serum-induced phosphorylation of mammalian hsp27 correlates with changes in its intracellular localization and levels of oligomerization. Eur J Biochem 1994; 221: 327-334

29 Monastyrskaya EA, Andreeva LV, Duchen MR, Wiegant F, Bayda LA, Manukhina EB, Malyshev IY. Adaptation to heat of cardiomyoblasts in culture protects them against heat shock: role of nitric oxide and heat shock proteins. Biochemistry (Mosc) 2003; 68: 816-821

$30 \mathrm{Kutuk} \mathrm{O}$, Poli G, Basaga H. Resveratrol protects against 4-hydroxynonenal-induced apoptosis by blocking JNK and c-JUN/AP-1 signaling. Toxicol Sci 2006; 90: 120-132

31 Jang YJ, Kim J, Shim J, Kim J, Byun S, Oak MH, Lee KW, Lee HJ. Kaempferol attenuates 4-hydroxynonenal-induced apoptosis in PC12 cells by directly inhibiting NADPH oxidase. J Pharmacol Exp Ther 2011; 337: 747-754

32 Jin HB, Yang YB, Song YL, Zhang YC, Li YR. Protective roles of quercetin in acute myocardial ischemia and reperfusion injury in rats. Mol Biol Rep 2012; 39: 11005-11009

33 Yan L, Zhang JD, Wang B, Lv YJ, Jiang H, Liu GL, Qiao Y, Ren M, Guo XF Quercetin inhibits left ventricular hypertrophy in spontaneously hypertensive rats and inhibits angiotensin II-induced H9c2 cells hypertrophy by enhancing PPAR- $\gamma$ expression and suppressing AP-1 activity. PLoS One 2013; 8: e72548

34 Khreiss T, József L, Hossain S, Chan JS, Potempa LA, Filep JG. Loss of pentameric symmetry of C-reactive protein is associated with delayed apoptosis of human neutrophils. J Biol Chem 2002; 277: 40775-40781

35 Li F, Wu JH, Wang QH, Shu YL, Wan CW, Chan CO, Kam-Wah Mok D, Chan SW. Gui-ling-gao, a traditional Chinese functional food, prevents oxidative stress-induced apoptosis in H9c2 cardiomyocytes. Food Funct 2013; 4: 745-753

36 Angeloni C, Spencer JP, Leoncini E, Biagi PL, Hrelia S. Role of quercetin and its in vivo metabolites in protecting H9c2 cells against oxidative stress. Biochimie 2007; 89: 73-82

37 de Bock M, DerraikJG, Brennan CM, Biggs JB, Morgan PE, Hodgkinson SC, Hofman PL, Cutfield WS. Olive (Olea europaea L.) leaf polyphenols improve insulin sensitivity in middle-aged overweight men: a randomized, placebo-controlled, crossover trial. PLoS One 2013; 8: e57622

38 Janjic $D$, Wollheim $C B$. Islet cell metabolism is reflected by the MTT (tetrazolium) colorimetric assay. Diabetologia 1992; 35: 482-485

39 Royall JA, Ischiropoulos $\mathrm{H}$. Evaluation of 2',7'-dichlorofluorescin and dihydrorhodamine 123 as fluorescent probes for intracellular $\mathrm{H}_{2} \mathrm{O}_{2}$ in cultured endothelial cells. Arch Biochem Biophys 1993; 302: 348-355

40 Salvioli S, Ardizzoni A, Franceschi C, Cossarizza A. JC-1, but not DiOC6(3) or rhodamine 123 , is a reliable fluorescent probe to assess delta psi changes in intact cells: implications for studies on mitochondrial functionality during apoptosis. FEBS Lett 1997; 411: 77-82 